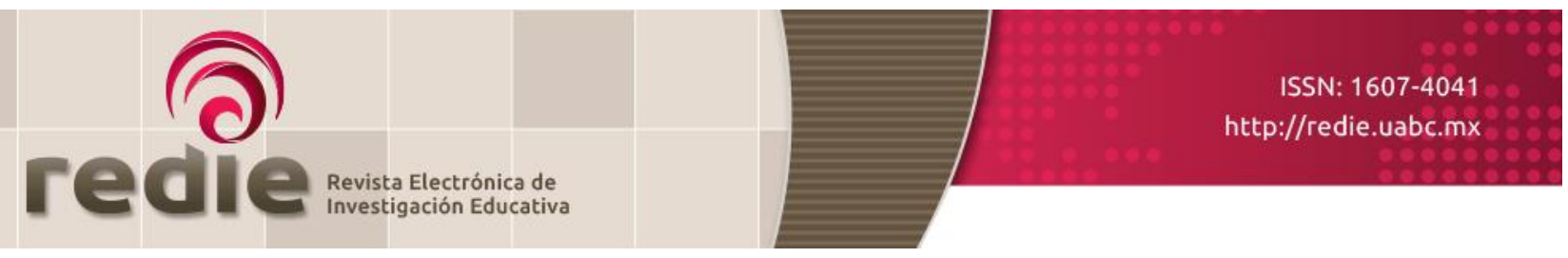

Vol. 22, 2020/e01

\title{
Validación de cuestionario sobre gestión del tiempo en universitarios mexicanos
}

\section{Validation of Time Management Questionnaire in Mexican College Students}

Gudiel Roblero (*) gudiel.roblero@ulv.edu.mx

(*) Universidad Linda Vista

(Recibido: 22 de agosto de 2017; Aceptado para su publicación: 15 de febrero de 2018)

Cómo citar: Roblero, G. (2020). Validación de cuestionario sobre gestión del tiempo en universitarios mexicanos. Revista Electrónica de Investigación Educativa, 22, e01, 1-11. https://doi.org/10.24320/redie.2020.22.e01.2136

\section{Resumen}

El uso que los estudiantes hacen del tiempo impacta en su desarrollo académico. La medición de la gestión del tiempo (GT) es un indicador que puede determinar varias áreas de la vida estudiantil. Se han diseñado instrumentos para medir el constructo señalado, sin embargo en México no se encontró validación alguna -por ello el presente estudio busca validar el cuestionario de la GT en universitarios mexicanos. Se realizó un estudio censal en el que participaron 289 estudiantes (191 hombres y 98 mujeres) de primer grado de dos universidades (una pública y una privada). Los datos se recolectaron mediante el instrumento validado en España y posteriormente en Venezuela, denominado Gestión de Tiempo en Estudiantes Universitarios (GTEU). Se usó el análisis factorial y el modelo de ecuaciones estructurales; la validación en México mostró semejanzas con las versiones española y venezolana en la agrupación de los factores y la carga factorial de cada ítem; se obtuvo una confiabilidad aceptable ( $a=$ $0.715, n=32)$ y un ajuste adecuado $\left(X^{2}=407.038 ; p=.278 ; \mathrm{gl}=391 ;\right.$ RMSEA $=.012 ; \mathrm{CFI}=.969$ y GFI $\left.=.92\right)$. Se concluyó que el GTEU es un instrumento que cuenta con las características psicométricas adecuadas para universitarios mexicanos.

Palabras clave: Validación, gestión del tiempo, universitarios.

\section{Abstract}

How students use their time affects their academic development. Time management (TM) may be a determining factor of various aspects of student life. Instruments have been designed to measure this construct but no validation was found in Mexico. Consequently, this study seeks to validate the TM questionnaire in Mexican college students. A census study was conducted with 289 undergraduate students (191 male and 98 female) from two universities (one public and one private). Data was collected using an instrument validated first in Spain, and subsequently in Venezuela, called "Time Management in College Students" (GTEU in Spanish). Factor analysis and structural equation modeling were used; validation in Mexico displayed similarities to the Spanish and Venezuelan versions in the clustering of factors and the factor loading of each item. The study revealed acceptable reliability $(a=0.715, n=32)$ 
and adequate goodness-of-fit $\left(\mathrm{X}^{2}=407.038 ; p=.278 ; \mathrm{gl}=391\right.$; RMSEA $=.012 ;$ CFI $=.969 \mathrm{y}$ GFI $\left.=.92\right)$. It is concluded that the GTEU possesses adequate psychometric properties for Mexican college students.

Keywords: Validity, time management, college students.

\section{Introducción}

Históricamente, la medición del uso del tiempo ha tenido un indudable valor para esclarecer y visibilizar de manera cuantitativa, la distribución de las tareas productivas de los individuos Hamui-Sutton et al., 2012). En educación, el tiempo es un factor clave en el aprendizaje (Monsalve et al., 2014), es un recurso limitado y su uso define las trayectorias profesionales y personales (Hamui-Sutton et al., 2012). Por otro lado, desde la perspectiva psicopedagógica se ha estudiado de manera eficientista y economicista (Vázquez, 2007).

El tiempo es el encargado de determinar lo que se ha de hacer en cada momento de la jornada escolar, plasmado en horarios y calendario que el alumnado asume sin cuestionar, y también es el mecanismo de control de profesorado por la administración educativa (Hargreaves, 1992).

Conceptualmente la Gestión del Tiempo (GT) se define como un proceso dirigido al establecimiento y logro de metas académicas definidas considerando el tiempo disponible y la verificación de sus usos (García-Ros y Pérez-González, 2012). Para tener una correcta GT, García y Santizo (2010) refieren que se deben tomar en cuenta los factores culturales, de estructura y dinámica organizacional, de comunicación, de tarea, centrados en equipos humanos y de educación de la organización a su entorno.

El constructo GT es multifactorial (Lay y Schouwenburg, 1993), para Broc (2011) incluye horario, planificación y manejo del propio tiempo de estudio. Está definido en términos de grupos de conductas para facilitar la productividad y aliviar el estrés (Lay y Schouwenburg, 1993), y constituye uno de los temas más tradicionales del aprendizaje y de estrategias de estudio, ocupando una posición central en los cursos y numerosos manuales sobre habilidades de estudio (García-Ros et al., 2004).

La GT tiene algunos beneficios que incluyen: reducir la procrastinación, tener más control, menos estrés, completar más tareas y disfrutar del tiempo libre (Nadinloyi et al., 2013); otros hallazgos empíricos relacionados concluyeron que los estudiantes de alto rendimiento tenían mejores habilidades de autorregulación del aprendizaje (Zimmerman y Martinez-Pons, 1990).

La GT también presenta efectos negativos como la deserción, se ha demostrado que los estudiantes que abandonan sus estudios a menudo tienen habilidades pobres en gestión de tiempo (Haarala-Muhonen et al., 2011, como se citó en Stelnicki et al., 2015), y puede reflejarse en ansiedad durante los exámenes y en niveles continuos de estrés (Akcoltekin, 2015).

Existen varios instrumentos sobre GT, los que más destacan por su uso en contextos universitarios son Time Structure Questionnaire (TSQ) (Bond y Feather, 1988), Time Management Behavior Questionnaire (TMBQ) (Macan et al., 1990) y Time Management Questionnaire (TMQ) (Britton y Tesser, 1991). En español, la literatura refiere la Gestión de Tiempo en Estudiantes Universitarios (cteu) de García-Ros y PérezGonzález (2012), quien retoma el instrumento ajustado por Macan (1994); por otro lado, Durán-Aponte y Pujol (2013) parten del trabajo realizado por García-Ros y Pérez-González (2012) y realizan una prueba psicométrica del instrumento en Venezuela (Monsalve et al., 2014).

La GT puede ser afectada por la cognición; además, los estudiantes deben ser capaces de manejar y regular su tiempo, su ambiente y contexto de estudio. El manejo del tiempo incluye un horario o programación horaria, planificación y manejo del propio tiempo de estudio. Esto incluye no sólo establecer períodos de tiempo para estudiar, sino también el uso efectivo de ese tiempo de estudio, así como establecer metas realistas (Broc, 2011). 
En México se hallaron dos estudios del uso del tiempo de la población en general, el realizado en 2009 incluye categorías compatibles con la International Classification of Activities for Time-Use Statistics (ICATUS) (Hamui-Sutton et al., 2012). Un estudio reciente en población con edad superior a 12 años revela que el promedio semanal que se dedica al aprendizaje y estudio es de 38.97 horas por semana (Sosa y Roman, 2015).

Los profesionales de la educación necesitan de instrumentos que permitan contrastar la validez y fiabilidad de este constructo educativo, pero no se han encontrado estudios previos en México que midan dicho constructo. Por otro lado, la deserción universitaria de estudiantes mexicanos es un factor más a considerar, la OCDE refiere que en 2015 México ocupó el primer lugar en abandono escolar universitario, y es durante el primer año cuando se produce la mayor cantidad de abandono (Gracia, 2015). En otro estudio se encontraron dos razones de abandono relacionadas con la GT: la dedicación y disciplina (5.8\%) y horarios complicados (16.4\%) (De Vries et al., 2011), el presente estudio pretende validar el cuestionario de la GT en universitarios mexicanos.

Se eligió al grupo de universitarios porque la transición del bachillerato al pregrado puede ser estresante para los nuevos estudiantes. La demanda de mayores niveles de independencia, la iniciativa y la autorregulación (Noel et al., 1985 y Bryde y Milburn, 1990, como se citó en Stelnicki et al., 2015) puede ser una situación especialmente difícil para los estudiantes al comenzar su viaje en la vida universitaria; además de que las validaciones hechas en España y Venezuela se realizaron con estudiantes de dicho nivel. En la tabla I se muestran los elementos considerados en las validaciones realizadas.

Tabla I. Validaciones de GTEU

\begin{tabular}{l|l|l}
\hline Instrumento & \multicolumn{2}{|c}{ Gestión del tiempo en estudiantes universitarios } \\
\hline Autores & García-Ros y Pérez-González (2012) & Durán-Aponte y Pujol (2013) \\
\hline Población & $\begin{array}{l}\text { Estudiantes españoles universitarios de primer } \\
\text { ingreso }\end{array}$ & $\begin{array}{l}\text { Estudiantes venezolanos universitarios de } \\
\text { primer ingreso }\end{array}$ \\
\hline Análisis & Análisis factorial de componentes principales & Análisis factorial de componentes principales \\
\hline Factores & $\begin{array}{l}4 \text { factores que explican el 42.93\% de la } \\
\text { varianza total }\end{array}$ & $\begin{array}{l}\text { 4 factores que explican el 34.35\% de la varianza } \\
\text { total }\end{array}$ \\
\hline
\end{tabular}

\section{Método}

Se realizó un estudio censal, cuantitativo, correlacional y transversal que incluyó a 289 estudiantes de dos universidades mexicanas, una privada (Universidad Linda Vista [ULV]), localizada en el sureño estado de Chiapas, y una pública (Universidad Autónoma de Nuevo León [UANL]), ubicada en el estado de Nuevo León, en el norte de México. De la ULV participaron 98 estudiantes (33.9\%) de segundo semestre en el período enero-junio de 2015, quienes cursaban las carreras de Enfermería, Nutrición, Educación, Administración, Teología y Sistemas Computacionales; mientras que de la UANL participaron 191 estudiantes (66.1\% de la muestra) de primer semestre de Ingeniería Civil en el período agosto-diciembre de 2015 . El $72.3 \%$ es de género masculino y el $27.7 \%$ femenino; a partir de los sujetos referidos se pretende extrapolar los resultados a estudiantes universitarios mexicanos. El tamaño de la muestra es suficiente para el análisis confirmatorio (Ramlall, 2017).

Рara cuidar los aspectos éticos se explicó a los estudiantes el objetivo del estudio y se solicitó participación voluntaria garantizando la confidencialidad de la información.

El instrumento utilizado se validó para la población española por García-Ros y Pérez-González (2012), y ajustado por Macan, del cual se derivaron 34 ítems; posteriormente se validó en Venezuela por DuránAponte y Pujol (2013), quedando conformado por 32 items -versión que se aplicó en el presente estudio. Los ítems en escala Likert van de 1 (Nunca) a 5 (Siempre). La tabla II muestra los 34 ítems de la versión española, y los 32 de la venezolana (del 1 a 32). Cabe precisar que se contó con la autorización de quienes validaron el instrumento (Rafael García-Ros para España, y Emilse Aponte para Venezuela). 
Tabla Il. Ítems de la versión en español del GTEU

\begin{tabular}{|c|c|c|}
\hline Ítems & Ven. & Méx. \\
\hline $\begin{array}{l}\text { 1. Cuando decido qué es lo que voy a intentar conseguir a corto plazo, tengo en cuenta también } \\
\text { mis objetivos a largo plazo. }\end{array}$ & $x$ & $x$ \\
\hline $\begin{array}{l}\text { 2. Cuando hago una lista de cosas a realizar, al final del día se me ha olvidado o la he dejado de } \\
\text { lado. }\end{array}$ & $x$ & $x$ \\
\hline 3. Llevo una libreta para apuntar notas e ideas. & $x$ & $x$ \\
\hline 4. Repaso mis objetivos para determinar si debo hacer cambios. & $x$ & $\mathrm{x}$ \\
\hline 5. Organizo mis actividades con al menos una semana de antelación. & $x$ & $x$ \\
\hline 6. Divido proyectos complejos y difíciles en pequeñas tareas más manejables. & $x$ & $\mathrm{x}$ \\
\hline 7. Al final de cada día dejo mi lugar de trabajo ordenado y bien organizado. & $x$ & $x$ \\
\hline 8. Establezco objetivos a corto plazo para lo que quiero conseguir en pocos días o semanas. & $\mathrm{x}$ & $\mathrm{x}$ \\
\hline 9. Tengo la sensación de controlar mi tiempo. & $x$ & $x$ \\
\hline $\begin{array}{l}\text { 10. Puedo encontrar las cosas que necesito más fácilmente cuando mi lugar de trabajo está "patas } \\
\text { arriba" y desordenado que cuando está ordenado y organizado. }\end{array}$ & $x$ & $x$ \\
\hline 11. Me marco fechas límite cuando me propongo realizar una tarea. & $x$ & $x$ \\
\hline 12. Escribo notas para recordar lo que necesito hacer. & $x$ & $\mathrm{x}$ \\
\hline 13. Tengo que emplear mucho tiempo en tareas sin importancia. & $x$ & $x$ \\
\hline $\begin{array}{l}\text { 14. El tiempo que invierto en gestionar el tiempo y organizar mi jornada de trabajo es tiempo } \\
\text { perdido. }\end{array}$ & $\mathrm{x}$ & $\mathrm{x}$ \\
\hline 15. Busco maneras de incrementar la eficacia con que realizo las actividades en mis estudios. & $x$ & $x$ \\
\hline $\begin{array}{l}\text { 16. Hago una lista de cosas que hacer cada día y pongo una señal al lado de cada tarea cuando la } \\
\text { he cumplido. }\end{array}$ & $x$ & $x$ \\
\hline \multicolumn{3}{|l|}{ 17. Encuentro difícil mantener un horario porque los demás me apartan de mi trabajo. } \\
\hline 18. Mis jornadas diarias son demasiado impredecibles para planificar y gestionar mi tiempo. & $x$ & $\mathrm{x}$ \\
\hline 19. Termino tareas de alta prioridad antes de realizar las menos importantes. & $x$ & $x$ \\
\hline 20. Llevo una agenda conmigo. & $\mathrm{x}$ & $\mathrm{x}$ \\
\hline 21. Cuando estoy desorganizado(a) soy más capaz de adaptarme a acontecimientos inesperados. & $x$ & $x$ \\
\hline 22. Repaso mis actividades diarias para ver dónde pierdo el tiempo. & $x$ & $\mathrm{x}$ \\
\hline 23. Mantengo un diario de las actividades realizadas. & $x$ & $\mathrm{x}$ \\
\hline 24.Tengo algunas de mis ideas más creativas cuando estoy desorganizado(a). & $\mathrm{x}$ & $\mathrm{x}$ \\
\hline \multicolumn{3}{|l|}{ 25. Durante un día de trabajo evalúo si estoy cumpliendo con el horario que he preestablecido. } \\
\hline 26. Utilizo un sistema de bandejas (archivos o carpetas) рага organizar la información. & $x$ & $\mathrm{x}$ \\
\hline $\begin{array}{l}\text { 27. Me doy cuenta de que estoy posponiendo las tareas que no me gustan, pero que son } \\
\text { necesarias. }\end{array}$ & $x$ & $\mathrm{x}$ \\
\hline $\begin{array}{l}\text { 28. Noto que puedo hacer un mejor trabajo si aplazo las tareas que no me gustan en lugar de } \\
\text { intentar hacerlas por orden de importancia. }\end{array}$ & $x$ & $\mathrm{x}$ \\
\hline 29. Establezco prioridades para determinar en qué orden haré las tareas cada día. & $\mathrm{x}$ & $\mathrm{x}$ \\
\hline $\begin{array}{l}\text { 30. Si estoy en algo y sé que voy a tener que esperar un tiempo, preparo alguna tarea para realizar } \\
\text { mientras tanto. }\end{array}$ & $\mathrm{x}$ & $\mathrm{x}$ \\
\hline $\begin{array}{l}\text { 31. Establezco bloques de tiempo en mis horarios para actividades que hago habitualmente } \\
\text { (compras, ocio, navegar por la web...) }\end{array}$ & $\mathrm{x}$ & $x$ \\
\hline 32. Encuentro lugares para trabajar donde puedo evitar interrupciones y distracciones. & $x$ & $\mathrm{x}$ \\
\hline 33. Subestimo el tiempo necesario para cumplimentar tareas. & & \\
\hline $\begin{array}{l}\text { 34. Cuando observo que contacto frecuentemente con alguien, apunto su nombre , dirección y } \\
\text { número de teléfono en un lugar especial. }\end{array}$ & & \\
\hline
\end{tabular}

El cuestionario validado en España está integrado por cuatro dimensiones, las que también son usadas para el estudio en Venezuela; los ítems se agruparon para la validación latinoamericana tal como se muestra en la tabla III. 
Tabla III. Dimensiones y agrupación de ítems de GTEU Venezuela

\begin{tabular}{l|l}
\hline \multicolumn{1}{c|}{ Dimensiones } & \multicolumn{1}{c}{ Ítems } \\
\hline Objetivos y prioridades & $5,8,9,11,19,22,25,29,30,31,32$ \\
Herramientas de gestión & $3,12,13,16,20,23$ \\
Preferencia por la desorganización & $2,7,10,14,17,18,21,24,27,28$ \\
Percepción del control & $1,4,6,15,26$ \\
\hline
\end{tabular}

Se empléo el análisis factorial confirmatorio, método que permite probar la teoría y hay evidencias de que las variables se integran en factores definidos (Henson y Roberts, 2006); por otro lado, los resultados analíticos pueden informar sobre las variables que se desean definir (Thomson y Daniel, 1996). Además de que es uno de los métodos estadísticos más usado para contrastar validez de constructo (Pérez-Gil et al., 2000) también evalúa el comportamiento de un mismo instrumento en numerosos estudios (Kimberlin y Winterstein, 2008) y replicar estudios es un fundamento de la ciencia (Henson y Smith, 2000 y Thompson, 1999, como se citó en Henson y Roberts, 2006).

Рara el análisis de los datos se usó SPSS v23 para el análisis factorial y AMOs 20 para los modelos de ecuaciones estructurales.

\section{Resultados}

Previo al análisis factorial se obtuvo el índice de adecuación muestral de Kaiser-Meyer-Olkin (KMO), que arrojó resultado de 0.774 y el test de esfericidad de Bartlett $\left(X^{2}=1846.88 ; p<0.000\right)$. Estos resultados indican que se cumple con los supuestos para el análisis factorial (Rodríguez y Mora, 2001). Al correr el análisis factorial se consideraron los autovalores mayores a 1.5, que explicaron el 34.92\% de la varianza total. La confiablidad del instrumento fue aceptable $(a=0.715, n=289)$, dado que el coeficiente de correlación fue superior a 0.70 (Litwin, 1995). En la tabla IV se puede notar que los ítems 16, 25 y 31 son los que más afectan para que la confiabilidad sea baja. 
Tabla IV. Correlación de ítems GTEu México

\begin{tabular}{c|c|c|c|c}
\hline ítem & $\begin{array}{c}\text { Correlación } \\
\text { elemento-total } \\
\text { corregida }\end{array}$ & $\begin{array}{c}\text { Correlación } \\
\text { múltiple } \\
\text { al cuadrado }\end{array}$ & $\begin{array}{c}\text { Alfa de Cronbach } \\
\text { si se elimina } \\
\text { el elemento }\end{array}$ & $\mathbf{n}$ \\
\hline 1 & 0.259 & 0.303 & 0.673 & 289 \\
\hline 2 & -0.265 & 0.299 & 0.702 & 289 \\
\hline 3 & 0.131 & 0.280 & 0.682 & 289 \\
4 & 0.371 & 0.314 & 0.666 & 289 \\
5 & 0.094 & 0.205 & 0.701 & 289 \\
6 & 0.322 & 0.220 & 0.669 & 289 \\
\hline 7 & 0.15 & 0.311 & 0.68 & 289 \\
8 & 0.377 & 0.351 & 0.666 & 289 \\
\hline 9 & 0.256 & 0.202 & 0.673 & 289 \\
10 & -0.017 & 0.303 & 0.693 & 289 \\
\hline 11 & 0.331 & 0.297 & 0.667 & 289 \\
\hline 12 & 0.363 & 0.419 & 0.664 & 289 \\
13 & 0.175 & 0.186 & 0.678 & 289 \\
\hline 14 & 0.051 & 0.235 & 0.686 & 289 \\
\hline 15 & 0.316 & 0.315 & 0.669 & 289 \\
\hline 16 & 0.391 & 0.339 & 0.661 & 289 \\
\hline 17 & 0.077 & 0.319 & 0.680 & 289 \\
\hline 18 & -0.002 & 0.331 & 0.690 & 289 \\
19 & 0.218 & 0.246 & 0.675 & 289 \\
\hline 20 & 0.314 & 0.384 & 0.667 & 289 \\
\hline 21 & 0.161 & 0.31 & 0.679 & 289 \\
\hline 22 & 0.359 & 0.309 & 0.665 & 289 \\
\hline 23 & 0.291 & 0.304 & 0.670 & 289 \\
\hline 24 & 0.19 & 0.328 & 0.677 & 289 \\
\hline 25 & 0.407 & 0.313 & 0.661 & 289 \\
\hline 26 & 0.205 & 0.220 & 0.676 & 289 \\
27 & 0.077 & 0.213 & 0.685 & 289 \\
\hline 28 & 0.113 & 0.202 & 0.683 & 289 \\
\hline 29 & 0.35 & 0.272 & 0.666 & 289 \\
\hline 30 & 0.387 & 0.315 & 0.663 & 289 \\
\hline 31 & 0.384 & 0.293 & 0.662 & 289 \\
\hline 32 & 0.095 & 0.211 & 0.684 & 289 \\
\hline & & & & \\
\hline
\end{tabular}

\subsection{Análisis factorial}

El análisis factorial mostró que los ítems se agrupan en cuatro factores. El factor uno incluyó todos los ítems de la validación venezolana excepto el 22, 31 y 32; a este factor se sumaron los ítems 1, 2, 4, 6,15 y 26; mientras que el segundo factor (gestión) incluye todos los de la versión citada y se agrega el 22 y 31. El tercer factor incluyó los ítems 7, 10, 14, 21, 24 y 32, mientras que el cuarto no incluyó ninguno de la versión venezolana y en éste se agruparon los ítems 17, 18, 27 y 28, que correspondían al tercer factor en la validación de Durán-Aponte y Pujol (2013). En la tabla V se presentan los resultados de la varianza explicada; para la versión mexicana se obtuvo menor porcentaje con relación a la española (42.93\%) y similar a la venezolana (35.45\%); la misma tendencia se manifestó con la fiablidad. 
Tabla V. Varianza explicada y fiabilidad

\begin{tabular}{l|c|c|c|c}
\hline \multirow{2}{*}{ Factor } & Varianza total aplicada & \multicolumn{3}{|c}{ Alfa de Cronbach } \\
\cline { 3 - 5 } & México & Esp. & Ven. & Méx. \\
\hline 1. Objetivos y prioridades & $15.36 \%$ & 0.840 & 0.781 & 0.723 \\
2. Herramientas de gestión & $8.04 \%$ & 0.790 & 0.748 & 0.666 \\
3. Percepción de control & $6.61 \%$ & 0.710 & 0.631 & 0.552 \\
4. Preferencia por desorganización & $4.91 \%$ & 0.720 & & 0.530 \\
\hline General & $34.92 \%$ & & & 0.715 \\
\hline
\end{tabular}

La tabla VI muestra que el ítem 19 (Termino tareas de alta prioridad antes de realizar las menos importantes) presentó la media más alta y el 23 (Mantengo un diario de las actividades realizadas) la más baja; respecto a la variabilidad, se encontró que el ítem 3 (llevo una libreta para apuntar notas e ideas) presentó más heterogeneidad y el 2 (Cuando hago una lista de cosas a realizar, al final del día se me ha olvidado o la he dejado de lado) fue el más homogéneo. El ítem 20 (Llevo una agenda conmigo) fue el que presentó la mayor carga factorial, mientras que el 26 (Utilizo un sistema de bandejas para organizar la información) presentó la menor.

Tabla VI. Descriptivos y solución factorial GTEU-MEX

\begin{tabular}{|c|c|c|c|c|c|c|c|c|}
\hline \multirow{2}{*}{ Ítem } & \multirow{2}{*}{ M } & \multirow{2}{*}{ DT } & \multicolumn{4}{|c|}{ Saturación factorial } & \multirow{2}{*}{ Comunalidad } & \multirow{2}{*}{$n$} \\
\hline & & & 1 & 2 & 3 & 4 & & \\
\hline 1 & 3.70 & 1.00 & .59 & & & & .37 & 289 \\
\hline 2 & 2.64 & .95 & & & & .38 & .36 & 289 \\
\hline 3 & 3.34 & 1.43 & & .39 & & & .29 & 289 \\
\hline 4 & 3.15 & 1.01 & .48 & & & & .31 & 289 \\
\hline 5 & 2.91 & 2.52 & & & & -.47 & .31 & 289 \\
\hline 6 & 3.13 & 1.09 & .51 & & & & .30 & 289 \\
\hline 7 & 3.65 & 1.14 & & & -.40 & & .27 & 289 \\
\hline 8 & 3.59 & .99 & .60 & & & & .37 & 289 \\
\hline 9 & 3.42 & 1.05 & .39 & & & & .21 & 289 \\
\hline 10 & 2.13 & 1.30 & & & .65 & & .44 & 289 \\
\hline 11 & 3.56 & 1.14 & .48 & & & & .28 & 289 \\
\hline 12 & 3.42 & 1.22 & & .64 & & & .52 & 289 \\
\hline 13 & 2.40 & 1.03 & & .45 & & & .30 & 289 \\
\hline 14 & 2.08 & 1.14 & & & .43 & & .27 & 289 \\
\hline 15 & 3.57 & 1.09 & .60 & & & & .38 & 289 \\
\hline 16 & 2.55 & 1.35 & & .57 & & & .39 & 289 \\
\hline 17 & 2.42 & 1.15 & & & & .58 & .48 & 289 \\
\hline 18 & 2.58 & 1.19 & & & & .52 & .45 & 289 \\
\hline 19 & 3.83 & 1.08 & .46 & & & & .31 & 289 \\
\hline 20 & 2.30 & 1.35 & & .67 & & & .53 & 289 \\
\hline 21 & 2.78 & 1.29 & & & .54 & & .35 & 289 \\
\hline 22 & 2.39 & 1.17 & & .45 & & & .30 & 289 \\
\hline 23 & 2.07 & 1.23 & & .64 & & & .46 & 289 \\
\hline 24 & 2.69 & 1.26 & & & .64 & & .43 & 289 \\
\hline 25 & 2.86 & 1.22 & .52 & & & & .33 & 289 \\
\hline 26 & 3.36 & 1.33 & .23 & & & & .11 & 289 \\
\hline 27 & 3.10 & 1.17 & & & & .51 & .27 & 289 \\
\hline 28 & 2.86 & 1.19 & & & .33 & & .20 & 289 \\
\hline 29 & 3.56 & 1.13 & .51 & & & & .30 & 289 \\
\hline 30 & 3.34 & 1.15 & .53 & & & & .33 & 289 \\
\hline 31 & 2.95 & 1.27 & & .40 & & & .35 & 289 \\
\hline 32 & 3.62 & 1.26 & & & -.41 & & .28 & 289 \\
\hline
\end{tabular}


En la tabla VII se muestra la correlación entre factores, se encontró que el factor 1 y 2 presenta correlación positiva significativa, de manera similar el factor 3 y 4 . Lo anterior indica que mientras mejor definición tengan los estudiantes de sus objetivos harán mayor uso de las herramientas de gestión. Además, se encontró que a mayor percepción de control se tenga, más preferencia por la desorganización de las tareas académicas.

Tabla IIII. Correlación de dimensiones

\begin{tabular}{l|c|c|c|c|c|c}
\hline \multicolumn{1}{c|}{ Factor } & \multirow{2}{*}{ M } & \multirow{2}{*}{ DT } & \multicolumn{4}{c}{ Correlación de Pearson } \\
\cline { 4 - 7 } & & & 1 & 2 & 3 & 4 \\
\hline 1. Objetivos y prioridades & 41.06 & 6.85 & 1 & & & \\
2. Herramientas de gestión & 21.40 & 5.73 & $.379 * *$ & 1 & & \\
3. Percepción de control & 13.64 & 3.52 & $.176^{* *}$ & .033 & 1 & \\
4. Preferencia por desorganización & 19.81 & 3.64 & -.086 & -0.16 & $.240^{* *}$ & 1 \\
\hline
\end{tabular}

** La correlación es significativa al nivel 0.01 (bilateral).

\subsection{Modelos de ecuaciones estructurales}

Se elaboraron modelos estructurales para identificar la relación entre las variables observables y las latentes. El primer modelo presentó diferencias en el ajuste como se muestra en los siguientes indicadores $X^{2}=840.850 ; p=.000 ; g l=458 ;$ RMSEA $=.054 ; \mathrm{CFI}=.732 ; \mathrm{CFI}=.843$. En este modelo los efectos directos entre todas las variables no tuvieron significatividad, así como la correlación de los constructos.

Para definir los indicadores de ajuste se buscó que cumplieran tres criterios: absoluto, comparativo y parsimonioso (Ruiz et al., 2016), y que los indicadores como X² sea no significativa (Verdugo, 2008); RMSEA fuera menor que 0.08 y CFI y GFI superiores a 0.9 y cercanos a 1 (Khine, 2013). Tras varios ensayos de bondad y ajuste se obtuvieron los siguientes indicadores: $\mathrm{X}^{2}=407.038 ; \rho=.278 ; \mathrm{gl}=391$; RMSEA $=.012$; CFI $=.969 \mathrm{y} \mathrm{GFI}=.92$; los cuales son satisfactorios y permiten inferir que existe un razonable ajuste entre el modelo y los datos utilizados en este trabajo (ver figura 1).

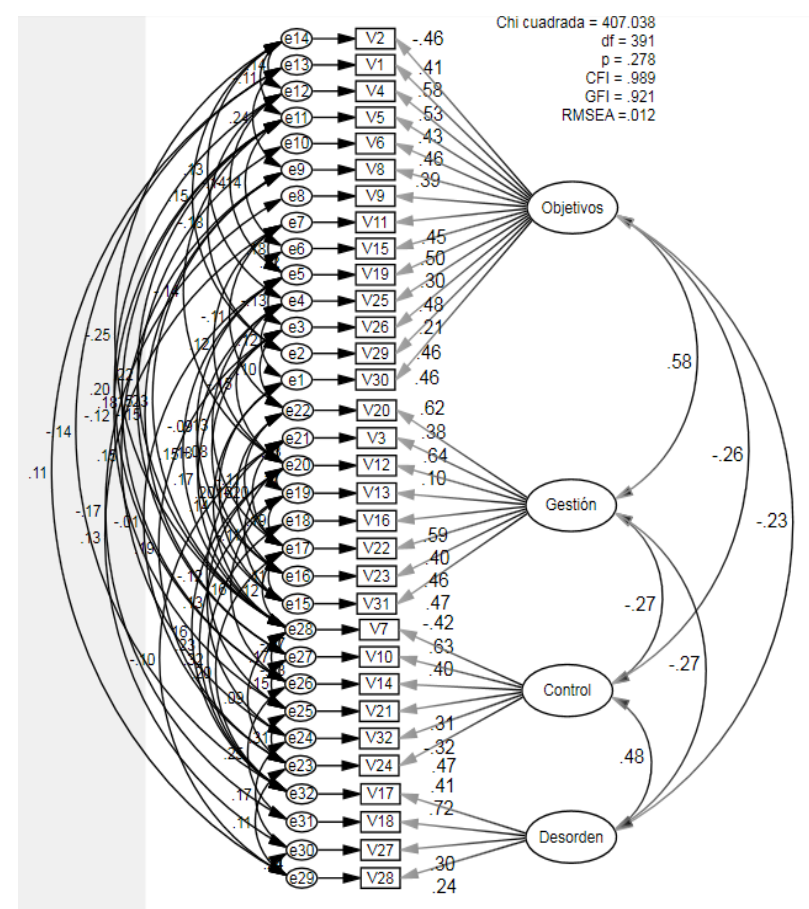

Figura 1. Modelo рага GTEU-MEX 


\section{Resultados y conclusiones}

El objetivo del estudio pretendió validar el cuestionario GTEU para México. Los resultados hallados difieren de las validaciones hechas en España y Venezuela. La adecuación muestral encontrada en España y Venezuela presenta KMO mayores que la versión mexicana; no obstante, es válida para proceder con el análisis factorial.

La muestra del caso México (289) es menor que la española (462) y mayor que la venezolana (209); respecto al género, en el estudio en cuestión la mayoría son varones (72.3\%), contrario a la muestra europea, en la que predominan las mujeres $(85.1 \%)$, mientras que en el estudio de Venezuela hubo equidad de género. Lo anterior puede ser una razón por la cual el estudio en México presentó menor confiabilidad, por tener una muestra más pequeña y porque las mujeres tienden a organizarse mejor que los varones. Al respecto, Todaro (2001) encontró que las mujeres fueron mejor valoradas en orden, disciplina y responsabilidad respecto a los varones.

En cuanto a los programas de estudio, en España los participantes pertenecían a dos programas, mientras que en Venezuela fueron estudiantes de diez programas diferentes y en el caso de México pertenecían a ocho diferentes carreras. Lo anterior puede ser otra de las explicaciones por las que se obtuvo una confiabilidad más baja en este estudio.

La varianza explicada del presente estudio (34.34\%) fue similar a la versión venezolana (35.35\%) y menor a la española (42.93\%).

Sobre la comunalidad, las cargas obtenidas fueron en promedio superiores (0.34) a las mínimas para los estudios empíricos (Tabachnick y Fidell, 2001, como se citó en Costello y Osborne, 2005); los ítems con la mayor y menor comunalidad en el estudio de México fueron el 20 (Llevo una agenda conmigo) y 28 (Noto que puedo hacer un mejor trabajo si aplazo las tareas que no me gustan en lugar de intentar hacerlas por orden de importancia); mientras que para el caso de España fueron el 26 (Tengo algunas de mis ideas más creativas cuando estoy desorganizado) y 28 (Utilizo un sistema de bandejas para organizar la información).

En lo referente a la correlación entre factores se encontró similitud en los correlatos de los factores 1 y 2 , 3 y 4 de la validación española y mexicana, obteniendo puntajes inferiores en el caso de México.

La confiabilidad obtenida en el presente estudio es inferior a la obtenida por García-Ross y PérezGonzález (2012) y Durán-Aponte y Pujol (2013); lo anterior se puede explicar porque este estudio se limitó a la validez factorial y no se realizó la de contenido.

El GTEU validado en España y replicado en Venezuela presentó semejanzas con la población mexicana; puede usarse para medir la gestión del tiempo en universitarios mexicanos y ser útil para investigaciones futuras, a pesar de que se obtuvo baja confiabilidad y menor varianza explicada es un indicador aceptable en las Ciencias Sociales; además, el modelo ecuaciones estructurales presentó un buen ajuste para la población mexicana.

\section{Agradecimientos}

Al Dr. Jaime Rodríguez Gómez por el análisis de ecuaciones estructurales; al Dr. Juan José Delgado por la recopilación y captura de los datos de la UANL, y a los estudiantes de Ciencias Administrativas del semestre 2015B de la ULV por la aplicación y captura de datos en ULV. 


\section{Referencias}

Akcoltekin, A. (2015). High school students' time management skills in relation to research anxiety, Educational Research and Reviews, 10(16), 2241-2249. http://doi.org/10.5897/ERR2015.2345

Broc, M. (2011). Voluntad para estudiar, regulación del esfuerzo, gestión eficaz del tiempo y rendimiento académico en alumnos universitarios. Revista de Investigación Educativa, 29(1), 171-185.

https://core.ac.uk/download/pdf/41554638.pdf

Costello, A. B. y Osborne, J. W. (2005). Best practices in exploratory factor analysis: four recommendations for getting the most from your analysis. Practical Assessment, Research \& Evaluation, 10(7). http://www.pareonline.net/pdf/v10n7.pdf

De Vries, W., Arenas, P., Muñoz, J. y Saldaña, I. (2011). ¿Desertores o decepcionados? Distintas causas para abandonar los estudios universitarios. Revista de La Educación Superior, 4(160), 29-49.

Durán-Aponte, E. y Pujol, L. (2013). Manejo del tiempo académico en jóvenes que inician estudios en la Universidad Simón Bolívar. Revista Latinoamericana de Ciencias Sociales, Niñez y Juventud, 11(1), 93-108. http://revistaumanizales.cinde.org.co/rlcsnj/index.php/Revista-Latinoamericana/article/view/835

García-Ros, R. y Pérez-González, F. (2012). Spanish version of the time management behavior questionnaire for university students. The Spanish Journal of Psychology, 15(3), 1485-1494.

http://doi.org/10.5209/rev SJOP.2012.v15.n3.39432

García-Ros, R., Pérez-González, F. e Hinojosa, E. (2004). Assessing time management skills as an important aspect of student learning. School Psychology International, 25(2), 167-183.

http://doi.org/10.1177/0143034304043684

García, J. y Santizo, J. (2010). Análisis de la reación entre la gestión del tiempo libre, el ocio y los estilos de aprendizaje. Revista Estilos de Aprendizaje, 3(5), 2-25.

Gracia, M. (22 de julio de 2015). Deserción Universitaria en México. Milenio.

http://www.milenio.com/firmas/maximiliano gracia hernandez/Desercion-universitaria-

Mexico 18 559324103.html

Hamui-Sutton, A., Aguirre-Hernández, R., Díaz-Villanueva, A., Ramírez-de la Roche, O. F. y Gómez-Lamont, D. S. (2012). Problemas académicos de estudiantes irregulares relacionados con el uso del tiempo en la Facultad de Medicina de la UNAM. Investigación en Educación Médica, 1(3), 107-113.

Hargreaves, A. (1992). El tiempo y el espacio en el trabajo del profesor. Revista de Educación, 298, 31-35.

Henson, R. K. y Roberts, J. K. (2006). Use of exploratory factor analysis in published research?: common errors and some comment on improved practice. Educational and Psychological Measurement, 66(3), 393416. http://doi.org/10.1177/0013164405282485

Khine, M. S. (2013). Application of structural equation modeling in educational research and practice. Sense Publishers.

Kimberlin, C. L., y Winterstein, a. G. (2008). Validity and reliability of measurement instruments used in research. American Journal of Health-System Pharmacy, 65(23), 2276-2284.

http://doi.org/10.2146/ajhp070364 
Lay, C. H. y Schouwenburg, H. (1993). Trait procrastination, time management, and academic behavior. Journal of Social Behavior y Personality, 8(4), 647.

https://www.researchate.net/publication/209836122 Trait procrastination time management and ac ademic behavior

Litwin, M. S. (1995). How to measure survey reliability and validity. Sage.

Monsalve, J. C., Romero, M. y Barberá, E. (2014). Evaluación de las competencias de gestión del tiempo en estudiantes de posgrado en modalidad virtual. Congreso Iberoamericano de Ciencia, Tecnología, Innovación y Educación, 1-13. http://www.oei.es/congreso2014/memoriactei/1327.pdf

Nadinloyi, K. B., Hajloo, N., Garamaleki, N. S. y Sadeghi, H. (2013). The study efficacy of time management training on increase academic time management of students. Procedia-Social and Behavioral Sciences, 84 , 134-138. http://doi.org/10.1016/j.sbspro.2013.06.523

Pérez-Gil, J. A., Chacón Moscoso, S. y Moreno Rodríguez, R. (2000). Validez de constructo: el uso de análisis factorial exploratorio-confirmatorio para obtener evidencias de validez. Psicothema, 12(Suplemento 2), 442-446.

Ramlall, I. (2017). applied structural equation modelling for researchers and practitioners : using r and stata for behavioural research.

https://search.ebscohost.com/login.aspx?direct=true\&db=nlebk\&AN=1423582\&lang=es\&site=ehost-live

Rodríguez, M-J. y Mora. R. (2001). Estadística Informática: casos y ejemplos con el SPSS. Universidad de Alicante.

Ruiz, M., Pardo, A. y San Martin, R. (2016). Sección Monográfica, 37(1), 52-61.

Sosa, V. y Roman, R. P. (2015). Participación y tiempo en actividades cotidianas de hombres y mujeres vinculados al mercado laboral en México. Sociedad y Economía, 29, 63-89.

Stelnicki, A. M., Nordstokke, D. W. y Saklofske, D. H. (2015). Who is the successful university student?? an analysis of personal resources. Canadian Journal of Higher Education, 45(2), 214-229.

Todaro, R., Godoy, L. y Abramo, L. (2001). Desempeño laboral de hombres y mujeres: opinan los empresarios Centro de Estudios de la Mujer (CEM). Desempeño laboral de hombres y mujeres. Cadernos Pagu, 1802(17), 197-236. http://www.scielo.br/pdf/cpa/n17-18/n17a08

Thomson, B. y Daniel, L. G. (1996). Factor analytic evidence for the construct validity ofscores: ahistor ical overview and some guidelines. Educational and Psychological Measurement, 56, 197-208

Vázquez, R. (2007). Reflexiones sobre el tiempo escolar. Revista Iberoamericana de Educación, 42, 1-11. http://dialnet.unirioja.es/servlet/articulo?codigo=2283788

Verdugo, M. A. (2008). Metodología en la investigación sobre discapacidad. Introducción al uso de las ecuaciones estructurales. Universidad de Salamanca.

Zimmerman, B. J. y Martinez-Pons, M. (1990). Student differences in self-regulated learning: relating grade, sex, and giftedness to self-efficacy and strategy use. Journal of Educational Psychology, 82, 51-59. https://psycnet.apa.org/record/1990-21082-001 